\title{
Computation and Characterization of Local Subfilter-Scale Energy Transfers in Atmospheric Flows ${ }^{\mathfrak{O}}$
}

\author{
Davide Faranda, ${ }^{\mathrm{a}, \mathrm{b}}$ Valerio Lembo, ${ }^{\mathrm{c}}$ Manasa Iyer, ${ }^{\mathrm{d}}$ Denis Kuzzay, ${ }^{\mathrm{e}}$ Sergio Chibbaro, ${ }^{\mathrm{d}}$ \\ FrANCOIS DAVIAUD, ${ }^{\mathrm{f}}$ AND BERENGERE DUBRULlE ${ }^{\mathrm{f}}$ \\ ${ }^{\text {a }}$ Laboratoire des Sciences du Climat et de l'Environnement, LSCE/IPSL, CEA-CNRS-UVSQ, \\ Université Paris-Saclay, Gif-sur-Yvette, France \\ ${ }^{\mathrm{b}}$ London Mathematical Laboratory, London, United Kingdom \\ ${ }^{\mathrm{c}}$ Meteorological Institute, University of Hamburg, Hamburg, Germany \\ d Sorbonne Université, UPMC Université Paris 06, CNRS UMR 7190, Institut Jean le Rond d'Alembert, Paris, France \\ ${ }^{\mathrm{e}}$ Université Lyon, Ens de Lyon, Université Claude Bernard, CNRS, Laboratoire de Physique, Lyon, France \\ ${ }^{\mathrm{f}} \mathrm{SPEC}, \mathrm{CEA}, \mathrm{CNRS}$, Université Paris-Saclay, CEA Saclay, Gif-sur-Yvette, France
}

(Manuscript received 12 April 2017, in final form 9 March 2018)

\begin{abstract}
Atmospheric motions are governed by turbulent motions associated to nontrivial energy transfers at small scales (direct cascade) and/or at large scales (inverse cascade). Although it is known that the two cascades coexist, energy fluxes have been previously investigated from the spectral point of view but not on their instantaneous spatial and local structure. Here, we compute local and instantaneous subfilter-scale energy transfers in two sets of reanalyses (NCEP-NCAR and ERA-Interim) in the troposphere and the lower stratosphere for the year 2005. The fluxes are mostly positive (toward subgrid scales) in the troposphere and negative in the stratosphere, reflecting the baroclinic and barotropic nature of the motions, respectively. The most intense positive energy fluxes are found in the troposphere and are associated with baroclinic eddies or tropical cyclones. The computation of such fluxes can be used to characterize the amount of energy lost or missing at the smallest scales in climate and weather models.
\end{abstract}

\section{Introduction}

In the classical turbulence phenomenology, valid in homogeneous flows, energy is injected at large scales, transferred downscale at a constant averaged rate $\varepsilon$ [Kolmogorov (1941) cascade], and dissipated at small scales by viscous effects (Frisch 1995). This phenomenology is based on the so-called Karman-HowarthMonin (KHM) equation, derived directly from the Navier-Stokes equation, that reads

$$
\frac{1}{2} \partial_{t} E+\varepsilon=-\frac{1}{4} \nabla_{\ell} \cdot\left\langle\delta \mathbf{u}(\delta u)^{2}\right\rangle+\nu \nabla_{\ell}^{2} E,
$$

\footnotetext{
Supplemental information related to this paper is available at the Journals Online website: https://doi.org/10.1175/JAS-D-170114.s1.
}

Corresponding author: Davide Faranda, davide.faranda@lsce. ipsl.fr where $u$ is the velocity field, $\nu$ is the molecular viscosity, the angle brackets indicate a statistical average, $\varepsilon$ is the mean nondimensional energy injection rate, $\delta \mathbf{u}=\mathbf{u}(\mathbf{x}+\ell)-\mathbf{u}(\mathbf{x})$ is the velocity increments over a distance $\ell$, and $E(\ell)=\left\langle(\delta u)^{2}\right\rangle / 2$ is a measure of the kinetic energy at scale $\ell$. The KHM equation can be seen as the counterpart in the physical space of traditional spectral energy budgets. In stationary situations, $(1 / 2) \partial_{t} E=0$, and the KHM equation describes how the injected energy $\varepsilon$ is split, at each given scale, into the two terms on the rhs: the "inertial term," $-(1 / 4) \nabla_{\ell} \cdot\left\langle\delta \mathbf{u}(\delta u)^{2}\right\rangle$, which comes from the nonlinear interactions, and the viscous term $\nu \nabla_{\ell}^{2} E$, which comes from the viscosity. This energy budget is valid at any scale, and depending on the value of the scale considered, either the inertial or the viscous term dominates: for scales larger than the Kolmogorov scale $\eta=\left(\nu^{3} / \varepsilon\right)$, the inertial term dominates, while the viscous term dominates for scales smaller than $\eta$. Overall, the picture is that of an energy cascade: as the scale is decreased, the energy injected at large-scale $\varepsilon$ is gradually transferred at a constant rate to the scales $\ell<\eta$, where it 
is dissipated into heat. The term $-(1 / 4) \nabla_{\ell} \cdot\left\langle\delta \mathbf{u}(\delta u)^{2}\right\rangle$ therefore describes an energy flux at the scale $\ell$, characterizing the cascade. It must be positive in order to describe a forward energy cascade, from large to small scales. This condition actually depends very much on the type of turbulence we are considering: for example, in 3D turbulence, the energy cascade is forward, while in 2D incompressible turbulence, it is backward, with formation of larger and larger structures. In any case, based on this equation, the observation of the existence of a constant energy flux and on a hypothesis of self-similarity, Kolmogorov was able to find out that the energy spectrum obeys a $k^{-5 / 3}$ law, corresponding to a forward energy cascade.

In the atmosphere, turbulence is much more complex than the homogeneous and isotropic one because of the influence of density stratification and rotation (Holton and Hakim 2012). Turbulence in such condition is known to develop a complex dynamics, with power-law energy spectra, as revealed by accurate numerical simulations and laboratory experiments (Levich and Tzvetkov 1985; Schertzer et al. 1997; Falkovich 1992; Pouquet and Marino 2013). Depending on the scale of the flow, energy transfers can be directed either toward smaller scales (direct cascade) or toward larger scales (inverse cascade; Bartello 1995). To date, there is in fact no general consensus about the direction of cascades in the atmosphere. Observed energy spectra in the troposphere and in the lower stratosphere (Nastrom and Gage 1985) exhibit a $k^{-5 / 3}$ law, generally connected to direct cascades, and/or $k^{-3}$ power laws, associated to an inverse energy cascade. The inverse cascade has been historically associated to the quasigeostrophic twodimensional dynamics induced by rotation (Charney 1971) and fed by baroclinic instability. Tung and Orlando (2003) simulated the Nastrom-Gage energy spectrum of atmospheric turbulence as a function of wavelength with a two-level quasigeostrophic model and were able to obtain both spectral behaviors with this simple dynamics. Kitamura and Matsuda (2006) analyzed the role of stratification and rotation in the generation of the cascades, observing that in experiments without planetary rotation, the obtained spectral slope was steeper and energy transfer to larger vertical wavenumbers was increased. Some theories of a mesoscale inverse cascade for stratified (not quasigeostrophic) turbulence were proposed by Gage (1979) and Lilly (1983), but these are no longer considered viable. According to Lindborg (2005), atmospheric mesoscale $-5 / 3$ energy spectra can be explained by the existence of a direct cascade arising in the limit of strong stratification, while the role of planetary rotation is to inhibit the cascade process at large scales, leading to an accumulation of kinetic energy and steepening of the kinetic energy spectrum at small wavenumbers. Evidence of the existence of a direct energy cascade comes from high-resolution direct numerical simulations of stratified flows (Lindborg 2006). They also suggest that the direction of the cascade may be crucially dependent on the ratio of the Brunt-Väisälä frequency to the rotation frequency.

A way to clarify the situation is to compute directly the energy fluxes. In the classical picture of turbulence, such energy transfers are related directly to the skewness of velocity increments $\left\langle\delta \mathbf{u}(\delta u)^{2}\right\rangle$, and the direction of the cascade is provided by the sign of this quantity (negative for direct cascade and positive for inverse cascade). This quantity, which is global, since it relies on a spatial average, has thus been used in the past to quantify the direction of the energy transfer. From the observed stratospheric third-order structure function, Lindborg and Cho (2001) argued that there is a forward energy cascade in the mesoscale range of atmospheric motions. In that study, the authors pointed out that for scales smaller than $100 \mathrm{~km}$, the statistical inhomogeneities can be neglected, while this assumption is not valid for larger scales.

Another approach relies on the spectral kinetic energy budgets (e.g., Augier and Lindborg 2013; Peng et al. 2015). Alternatively, one may compute energy budget in the physical space by considering appropriate generalization of the Karman-Howarth-Monin equation to include the influence of rotation and stratification. To this aim, Augier et al. (2012) recently considered a set of primitive equations for incompressible, nondiffusive, and inviscid stably stratified fluid in the Boussinesq approximation in order to account for both the kinetic energy (KE) and available potential energy (APE) in a modified version of the KHM equation. The primitive equations for the rotating stratified Boussinesq fluid were written as

$$
\begin{aligned}
\nabla \cdot \mathbf{u} & =0, \\
\partial_{t} \mathbf{u}+(\mathbf{u} \cdot \nabla) \mathbf{u}+2 \mathbf{\Omega} \times \mathbf{u} & =-\nabla p+b \mathbf{e}_{z}+\nu \Delta \mathbf{u}, \\
\partial_{t} b+(\mathbf{u} \cdot \nabla) b & =-N^{2} u_{z}+\kappa \Delta b,
\end{aligned}
$$

where $\mathbf{u}$ is the velocity field, $u_{z}$ its vertical component, $\mathbf{\Omega}$ the rotation rate, $p$ the rescaled pressure, $\nu$ and $\kappa$ the viscosity and diffusivity, $N=\sqrt{-\left(g / \rho_{0}\right)(d \bar{\rho} / d z)}$ the constant Brunt-Väisälä frequency, $\mathbf{e}_{z}$ the vertical unit vector, $b=-\rho^{\prime} g / \rho_{0}$ the buoyancy perturbation, $g$ the acceleration due to gravity, $\rho_{0}$ a reference density, $\bar{\rho}(z)$ the mean profile, and $\rho^{\prime}$ a density perturbation. The resulting generalized KHM equation was written as

$$
\frac{1}{2} \partial_{t} E+\varepsilon=-\frac{1}{4} \nabla_{\ell} \cdot\left\langle\delta \mathbf{u}\left[(\delta u)^{2}+\frac{(\delta b)^{2}}{N^{2}}\right]\right\rangle \equiv \nabla \cdot \mathbf{J},
$$


where $E$ is now

$$
E=\frac{1}{2}\left\langle(\delta u)^{2}\right\rangle+\left\langle(\delta b)^{2} / N^{2}\right\rangle,
$$

and we have omitted contributions due to viscosity and diffusivity. Note that the rotation does not enter explicitly into this energy budget because the Coriolis force is perpendicular to the flow. It enters implicitly into the energy budget through third-order correlations, which can be shown to depend explicitly on the rotation rate (Campagne 2015). This shows that the energy flux $\mathbf{J}$ is made of a KE flux and an APE flux and formalizes the Lorenz energy cycle (LEC) description of the atmospheric energy budget (Lorenz 1955) via the conversion of APE into KE and then into dissipative heating. The KE and APE fluxes can have different direction so that the resulting energy flux can be positive or negative depending on the scale, isotropy, or stratification, and the corresponding direction of the energy cascade is hard to predict (Lovejoy and Schertzer 2010). These approaches nevertheless only provide a global estimate of the energy transfers in space, so that one cannot connect them with observed coherent structures observed in the atmosphere. One improvement of our understanding of energy transfers would therefore require their local in space and time estimates at any given scale.

This is now possible through an important breakthrough made by Duchon and Robert (2000), who reformulated the energy budget of the Navier-Stokes equations into a form allowing for the definition of energy transfers local in space and time and valid for any geometry, including when strong inhomogeneity and anisotropy are present. Its ability to provide interesting information about energy transfers at a given scale $\ell$ has been so far exploited in the experimental setup of the von Kármán swirling flow to measure the scale-to-scale energy transfers and nonviscous energy dissipation (Kuzzay et al. 2015; Saw et al. 2016). The Duchon and Robert indicator requires only the 3D velocity fields and provides, for each instant, 3D maps of the subfilter energy transfers at a scale $\ell$. The interest of this formulation is that it is devoid of any adjustable parameters unlike, for example, local estimates of energy budgets based on LES methods (Kuzzay et al. 2015). In this work, we adapt the definition of such an indicator to the atmospheric dynamics providing the first local maps of subfilter-scale energy transfers without any adjustable parameter. The goal of this work is (i) to identify and characterize the atmospheric motions responsible for large energy transfers and (ii) to compute global temporal and spatial averages and assess whether the reanalyses over- or underrepresent the energy fluxes.
The paper is structured as follows. After presenting the indicator, we will study these transfers in the National Centers for Environmental Prediction-National Center for Atmospheric Research (NCEP-NCAR) reanalysis and ERA-Interim - to study the sensitivity of the results to the resolution-for the year 2005. This year is ideal, as it does not correspond to major ENSO events or volcanic eruptions. We investigate (i) the vertical and horizontal global averages and (ii) the distribution of energy transfers at different scales. Results are displayed in arbitrary units, but in the same scale, for the NCEP-NCAR reanalysis and ERA-Interim. Finally, we discuss the implications of our results on a theoretical and practical level.

\section{Methods}

For any solutions of the Navier-Stokes equations, Duchon and Robert (2000) defined energy transfers in a fluid at an arbitrary scale $\ell$ using a local energy balance equation:

$$
\begin{aligned}
\partial_{t} & E^{\ell}+\partial_{j}\left[u_{j} E^{\ell}+\frac{1}{2}\left(u_{j} \hat{p}+\hat{u}_{j} p\right)+\frac{1}{4}\left(\widehat{u^{2} u_{j}}-\widehat{u^{2}} u_{j}\right)-\nu \partial_{j} E^{\ell}\right] \\
& =-\nu \partial_{j} u_{i} \partial_{j} \hat{u}_{i}-\mathcal{D}_{\ell}
\end{aligned}
$$

where $u_{i}$ are the components of the velocity field, $p$ is the pressure, $\hat{u}$ and $\hat{p}$ are their coarse-grained components at scale $\ell, E^{\ell}=\hat{u}_{i} u_{i} / 2$ is the kinetic energy per unit mass at scale $\ell$ (such that $\lim _{\ell \rightarrow 0} E^{\ell}=u^{2} / 2$ ), and $\mathcal{D}_{\ell}$ is expressed in terms of velocity increments $\delta \mathbf{u}(\mathbf{r}, \mathbf{x}) \stackrel{\text { def }}{=} \mathbf{u}(\mathbf{x}+\mathbf{r})-\mathbf{u}(\mathbf{x}) \equiv \delta \mathbf{u}(\mathbf{r}) \quad$ (the dependence on $\ell$ and $\mathbf{x}$ is kept implicit) as

$$
\mathcal{D}_{\ell}(\mathbf{u})=\frac{1}{4 \ell} \int_{\mathcal{V}} d \mathbf{r}\left(\nabla G_{\ell}\right)(\mathbf{r}) \cdot \delta \mathbf{u}(\mathbf{r})|\delta \mathbf{u}(\mathbf{r})|^{2},
$$

where $G$ is a smooth filtering function, nonnegative, spatially localized and such that $\int d \mathbf{r} G(\mathbf{r})=1$ and $\int d \mathbf{r}|\mathbf{r}|^{2} G(\mathbf{r}) \approx 1$. The function $G_{\ell}$ is rescaled with $\ell$ as $G_{\ell}(\mathbf{r})=\ell^{-3} G(\mathbf{r} / \ell)$. The choice of $G$ slightly determines the local energy budget in the sense that different choices of $G$ may result in a different level of kinetic or potential energy at a given scale $\ell$, as well as a different repartition of injected energy between the kinetic and potential part. In that respect, the local energy budget is $G$ sensitive, and one should choose the filter that is more appropriate to boundary conditions and symmetry of the equations. However, we have checked on numerical simulations of the incompressible Navier-Stokes equations that the spatial average of the different terms is not 
sensitive to the choice of $G$. Moreover, as shown in Duchon and Robert (2000), the choice of $G$ has no impact on the value of $\mathcal{D}_{\ell}$, in the limit $\ell \rightarrow 0$, as long as it satisfies the properties specified previously. So we expect the $G$ sensitivity of the analysis to decrease with scale.

In the following, we choose a spherically symmetric function of $x$, which has a Gaussian shape, because this filter function occurs naturally in any observational or experimental flow measurement or in LES. The corresponding energy budget will then have a straightforward physical meaning. This filter is given by

$$
G_{\ell}(\mathbf{r})=\frac{1}{N} \exp \left\{-1 /\left(1-\left[\mathbf{r} /(2 \ell)^{2}\right]\right)\right\}
$$

where $N$ is a normalization constant such that $\int d^{3} \mathbf{r} G(\mathbf{r})=1$. As we show later, results with this filter for two different data reanalyses are consistent with each other at different resolutions. This makes us confident that our results are robust.

As noticed by Duchon and Robert, the average of $\mathcal{D}_{\ell}(\mathbf{u})$ can be viewed as a weak form of the transfer term $-(1 / 4) \nabla_{\ell} \cdot\left\langle\delta \mathbf{u}(\delta u)^{2}\right\rangle$ in the anisotropic version of the KHM equation [Eq. (1)], the divergence being taken not on the term itself but instead on the test function $G_{\ell}$. Therefore, $\mathcal{D}_{\ell}(\mathbf{u})$ is a local version (no average is taken) of the energy transfer term of the KHM equation. By construction, $\mathcal{D}_{\ell}(\mathbf{u})$ represents the amount of energy transferred at the scale $\ell$ by the inertial term. For scales larger than the Kolmogorov scale (which is very small in atmospheric flow), this term provides most of the energy transfer, since the viscous contribution is negligible. Its sign provides the direction of the fluxes in the scale space: a positive sign implies transfer toward the scales smaller than $\ell$.

By construction, the intrinsic weak formulation of $\mathcal{D}_{\ell}(\mathbf{u})$ makes it less sensitive to noise than classical gradients or even than the usual KHM relation: indeed, the derivative in scale is not applied directly to the velocity increments but rather on the smoothing function, followed by a local angle averaging. This guarantees that no additional noise is introduced by the procedure. Additionally, the noise coming from the estimate of the velocity is naturally averaged out by the angle smoothing, as shown in Kuzzay et al. (2015). In the same study, the authors argued that the Duchon and Robert approach was a better alternative to the widespread LES-based method for the computation of energy fluxes, since it relies on very few arbitrary hypotheses. Experimentally, in the von Kármán setup, the Duchon-Robert (DR) formula provided a better estimate of the energy dissipation than an LES method: in particular, estimates of the injected and dissipated powers were within $20 \%$ of the measured value using the LES-particle image velocimetry (PIV) method, whereas they reached
$98 \%$ of the actual dissipation rate of energy with the DR formula (Kuzzay et al. 2015).

This approach for atmospheric dynamics requires taking into account density stratification and considering Boussinesq equations instead of Navier-Stokes equations. We have adapted the Duchon-Robert formalism to the Boussinesq equations. The equation for the kinetic energy is simply restated as

$\partial_{t} E^{\ell}+\nabla \cdot \mathbf{J}_{K}^{\ell}=-\nu \partial_{j} u_{i} \partial_{j} \hat{u}_{i}-\mathcal{D}_{\ell}+\frac{1}{2}\left(b \hat{u}_{z}+\hat{b} u_{z}\right)$.

Using the point-split buoyancy perturbation as fundamental variable, we can then obtain an equation related to the local variance of the buoyancy perturbation (details are given in the appendix):

$\partial_{t} E_{T}^{\ell}+\nabla \cdot \mathbf{J}_{T}^{\ell}=-\mathcal{D}_{\ell}^{T}-\frac{1}{2}\left(b \hat{u}_{z}+\hat{b} u_{z}\right)-\kappa \partial_{j} \hat{b}_{j} b / N^{2}$,

where $E_{T}^{\ell}=\hat{b} b /\left(2 N^{2}\right)$ is the available potential energy at scale $\ell$ and $\mathcal{D}_{\ell}^{T}$ is expressed in terms of the increments $\delta b(\mathbf{r}, \mathbf{x}) \stackrel{\text { def }}{=} b(\mathbf{x}+\mathbf{r})-b(\mathbf{x}) \equiv \delta b(\mathbf{r})$ (the dependence on $\ell$ and $\mathbf{x}$ is kept implicit in the equations) as

$$
\mathcal{D}_{\ell}^{T}=\frac{1}{4 \ell} \int_{\mathcal{V}} d \mathbf{r}\left(\nabla G_{\ell}\right)(\mathbf{r}) \cdot \delta \mathbf{u}(\mathbf{r})|(\delta b)|^{2} / N^{2} .
$$

Considering now that the energy for stratified flows is given by expression (4), we can sum Eqs. (8) and (9) to get the total local energy balance:

$\partial_{t} E^{\ell}+\nabla \cdot \mathbf{J}^{\ell}=-\mathcal{D}_{\ell}(\mathbf{u}, b)-\nu \partial_{j} \hat{u}_{i} \partial_{j} u_{i}-\kappa \partial_{j} \hat{b} \partial_{j} b / N^{2}$,

where

$$
\mathbf{J}^{\ell}=\mathbf{J}_{K}^{\ell}+\mathbf{J}_{T}^{\ell}
$$

is the spatial energy flux and

$\mathcal{D}_{\ell}(\mathbf{u}, b)=\frac{1}{4 \ell} \int_{\mathcal{V}} d \mathbf{r}\left(\nabla G_{\ell}\right)(\mathbf{r}) \cdot \delta \mathbf{u}\left[(\delta u)^{2}+\frac{(\delta b)^{2}}{N^{2}}\right]$,

is the total local scale-to-scale energy flux. It is easy to see that the average of $\mathcal{D}_{\ell}(\mathbf{u}, b)$ is a weak formulation of the energy transfer terms of the generalized KHM equation of Augier et al. [2012, Eq. (3)]. The DR indicator $\mathcal{D}_{\ell}(\mathbf{u}, b)$ is thus a local energy transfer term, which can be split into a kinetic (dynamical) part $\mathcal{D}_{\ell}(\mathbf{u})$ (the original DR indicator) and a potential (thermodynamic) part (the remaining part, implying the field $b$ ). To easily implement the expression of $\mathcal{D}_{\ell}(\mathbf{u}, b)$ in climate models, the buoyancy parameter has been rewritten as a function of 
temperature $T$ using the equation of state for dry air: $\delta b=-\left[\delta p /\left(\rho_{0} R\right)\right](1 / \delta T)$, where $\rho_{0}$ is a reference density at surface pressure and $\delta p$ is a pressure horizontal perturbation, which is set to be about $1 \mathrm{hPa}(100 \mathrm{~km})^{-1}$. Furthermore, in Eq. (13), we set a constant Brunt-Väisälä frequency, amounting to $1.2 \times 10^{-2} \mathrm{~s}^{-1}$ (Holton and Hakim 2012). In this way, the computation of $\mathcal{D}_{\ell}(\mathbf{u}, b)$ only requires the numerical $3 \mathrm{D}$ velocity $\mathbf{u}$ and $T$ fields.

The sign and geometry of the zones associated with high and low values of $\mathcal{D}_{\ell}(\mathbf{u}, b)$ will then provide interesting information about the dynamics of the energy exchange in the atmosphere. For example, a study of the occurrence of high and low values of $\mathcal{D}_{\ell}(\mathbf{u})$ in the von Kármán swirling flow has revealed that such events are associated with well-defined, characteristic geometry of the velocity field (Saw et al. 2016). For the kinetic (dynamical) part, positive values of $\mathcal{D}_{\ell}(\mathbf{u})$ are measured whenever there is a strong convergence of the flow. Divergent flows are instead associated with negative values of $\mathcal{D}_{\ell}(\mathbf{u})$, and they point to injection of energy from the subfilter scales. This simple description is not valid anymore when we also consider the potential (thermodynamic) component. For all these reasons, we cannot reduce the computation of $\mathcal{D}_{\ell}(\mathbf{u})$ to only that of the divergence/vorticity.

\section{Analysis}

For this study, outputs of ERA-Interim and NCEPNCAR Reanalysis-1 have been used. ERA-Interim is a modern-generation reanalysis with a much-higherresolution model. NCEP-NCAR Reanalysis-1 was pioneering when it was developed but is run at a comparatively low resolution and does not take advantage of as many observations as ERA-Interim.

ERA-Interim is the currently operational reanalysis product at the European Centre for Medium-Range Weather Forecasts (ECMWF; Dee et al. 2011). Released in 2007, it provides reanalyzed data from 1979 to the present, stored at an original T255 spectral resolution (about 80-km horizontal resolution), with 60 vertical hybrid model levels. A 12-h four-dimensional variational (4D-Var) data assimilation is adopted. As a forecast model, the Integrated Forecast System (IFS), cy31r2 release, is used, fully coupling modules for the atmosphere, ocean waves, and land surface. Sea surface temperatures (SSTs) and sea ice concentration (SIC) are ingested as boundary conditions and interpolated on a reduced Gaussian grid as needed. In our case, zonal, meridional, and vertical wind components are considered at a $0.75^{\circ} \times 0.75^{\circ}$ horizontal resolution over 12 pressure levels between 1000 and $100 \mathrm{hPa}$. A 12-h time step is considered. Known problems concerning these datasets are the lack of dry mass conservation (Berrisford et al. 2011) and the slight asymmetry between evaporation and precipitation (Dee et al. 2011). The turbulent fluxes are based on the tiled ECMWF scheme for surface exchanges over land (Viterbo and Beljaars 1995; Viterbo and Betts 1999). Each grid box is divided into up to six fractions (over land) depending on the type of surface, having different transfer coefficients based on a Monin-Obukhov formulation. Similarly, over oceans, two different coefficients are used for stable and unstable conditions (Beljaars 1995).

NCEP-NCAR Reanalysis-1 has been developed in a joint effort by NCEP and NCAR (Kalnay et al. 1996). The simulation has been operational since January 1995, covering a period from 1948 to the present. Data assimilation is performed via a $3 \mathrm{D}$ variational scheme (Parrish and Derber 1992). It features a T62 spectral resolution, corresponding to a $2.5^{\circ} \times 2.5^{\circ}$ horizontal grid (about 200-km horizontal resolution), with 28 sigma levels. Most of the major physical processes involving the climate system are parameterized. SST, SIC, snow cover, albedo, soil wetness, and roughness length are ingested as boundary conditions. Data are archived at an original 6-h time step, and such a temporal resolution is retained for our analysis. The atmospheric model that provides the NCEP-NCAR reanalysis data uses bulk aerodynamic formulas to estimate the turbulent fluxes, with exchange coefficients depending on empirical profiles extending the Monin-Obukhov similarity relationship (Miyakoda and Sirutis 1986). For more details on the comparison between different subgrid parameterization of surface fluxes, refer to Brunke et al. (2011).

\section{Analysis of local energy transfers}

\section{1) ONE YEAR'S AND SEASONS' AVERAGES OF LOCAL ENERGY TRANSFERS}

We begin the analysis by studying the latitudinal averages and the spatial features of the DR indicator for both ERA-Interim and the coarser NCEP-NCAR reanalysis. To enable comparison between the two datasets, one has to choose the analysis length larger than the resolution scale of NCEP-NCAR $(200 \mathrm{~km})$ since going below the resolution size introduces spurious effects dependent on the filter design. On the other hand, since we want to obtain as much detail as possible, we have to choose the smallest scale consistent with those requirements. Here, we thus adopt a scale of $\ell=220 \mathrm{~km}$, this scale being the smallest that provides reliable estimates of the DR indicator. A further discussion of the dependence of the results with scale is done in section $3 \mathrm{a}(3)$.

Results obtained for both reanalyses are consistent with each other, as can be checked from Fig. 1 (ERAInterim) and Fig. 2 (NCEP-NCAR). The gross features 

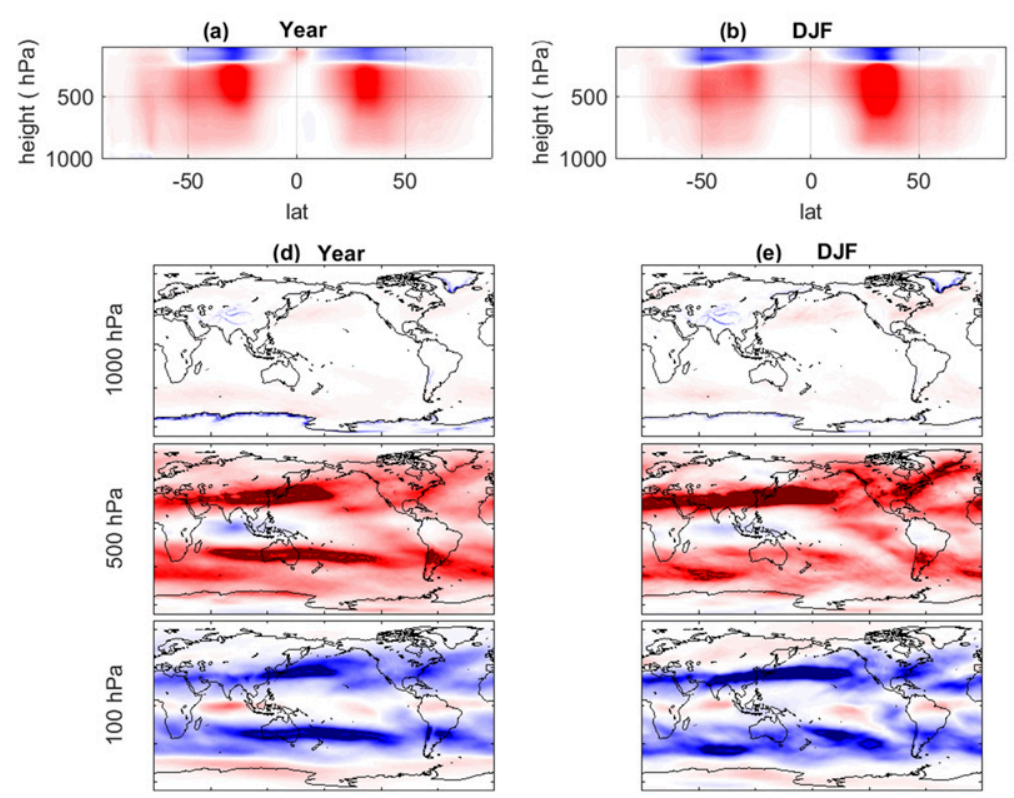
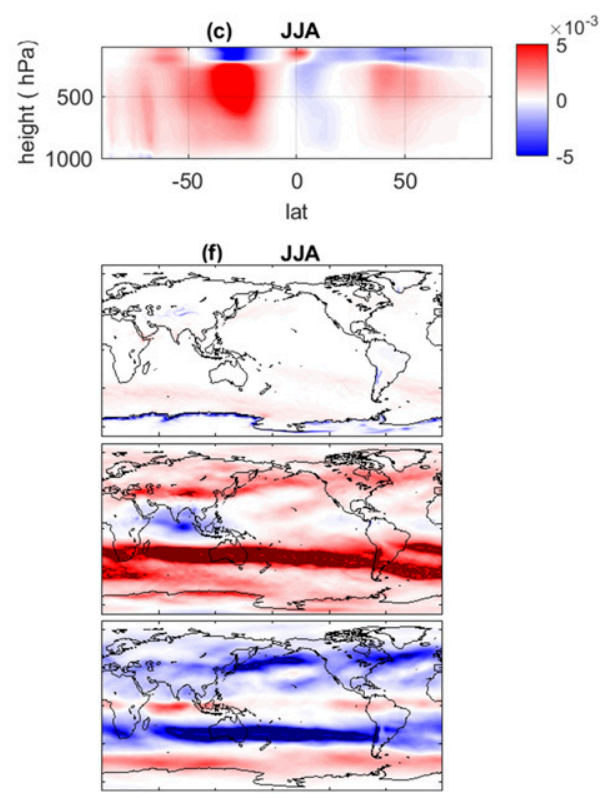

FIG. 1. Distribution of $\mathcal{D}_{\ell}(\mathbf{u}, b)$ for $\ell=220 \mathrm{~km}$ and ERA-Interim. (a)-(c) Longitudinal averages, (d)-(f) maps at three different fixed heights: 1000, 500, and $100 \mathrm{hPa}$. (a),(d) Yearly averages, (b),(e) winter averages, and (c),(f) summer averages.

do not depend on whether one undertakes an annual average (Figs. 1a,d and 2a,d) or seasonal average (Figs. 1b,c,e,f and 2b,c,e,f): in Figs. 1a-c and 2a-c, which show height dependence of the longitudinally averaged $\left\langle\mathcal{D}_{\ell}(\mathbf{u}, b)\right\rangle_{\text {long }}$, one observes the total local energy transfers $\mathcal{D}_{\ell}(\mathbf{u}, b)$ are mostly positive in the troposphere, about zero at the tropopause, and negative in the lower stratosphere. By looking at cuts at different pressure levels, one can more precisely evidence the spatial distribution of the yearly and seasonal averages of $\left\langle\mathcal{D}_{\ell}(\mathbf{u}, b)\right\rangle_{\text {time }}$. Close to the ground $(P=1000 \mathrm{hPa})$, the DR indicator is approximately zero except in proximity of sharp elevation gradients (Antarctica coasts and Himalaya, Greenland, and Andes mountain ranges). By splitting the local energy transfers into their kinetic $\left\langle\mathcal{D}_{\ell}(\mathbf{u})\right\rangle_{\text {time }}$ and thermodynamic parts $\left\langle\mathcal{D}_{\ell}^{T}\right\rangle_{\text {time }}$ (Figs. S1-S4 of the supplemental material), one can see that this effect is mostly due to the density fluctuations (i.e., the thermodynamic component of the DR indicator) that produce these negative fluxes.

In the middle troposphere $(P=500 \mathrm{hPa})$, the behavior of $\mathcal{D}_{\ell}(\mathbf{u}, b)$ is associated with the jet stream, since the most intense positive patterns are observed in winter for the Northern Hemisphere and in summer for the Southern Hemisphere. In the lower stratosphere $(P=$ $100 \mathrm{hPa}), \mathcal{D}_{\ell}(\mathbf{u}, b)$ is negative in midlatitudes and become slightly positive in polar regions and in the intertropical convergence zone. Overall, the splitting between the kinetic and thermodynamic components detailed in the supplemental material suggests that the dynamical component dominates with respect to the thermodynamic one, although the DR thermodynamic contributions are significant, especially in the proximity of the ground.

\section{2) CORRELAtion WITH ENERGY SPECTRUM}

The above result shows that the kinetic energy flux is globally positive in the troposphere, indicating a direct kinetic energy cascade, while they are negative in the lower stratosphere, indicating an inverse kinetic energy cascade. Our results are therefore consistent with those found by Peng et al. (2015), who also found upscale transfer in the lower stratosphere at outer mesoscale length scales and downscale transfers at scales smaller than 360 (KE) or $200 \mathrm{~km}$ (APE).

To get some insight on these cascades, we have further computed the kinetic horizontal energy spectra where $k$ is the inverse of the wavelength from the horizontal velocity fields at different pressure levels in the two reanalyses. They are reported in Fig. 3. One sees that for $P \leq 500 \mathrm{hPa}$ (corresponding to the stratosphere), the energy spectrum is mostly scaling like $k^{-3}$, while for $P \geq$ $500 \mathrm{hPa}$ (middle troposphere), the energy spectrum scales like $k^{-5 / 3}$, at least for scales larger than $\ell=220 \mathrm{~km}$-in agreement with the Nastrom-Gage spectrum in the lower stratosphere at scales between $10^{3}$ and $10^{2} \mathrm{~km}$. In the ERA-Interim data, the spectrum steepens below this scale and is closer to $k^{-2}$. These values are however to be taken with caution, since our resolution does not allow one to distinguish clearly 


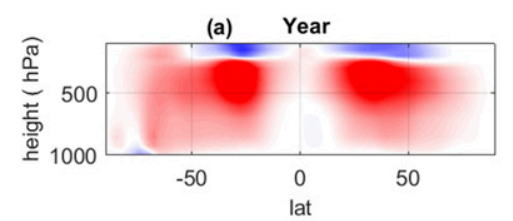

(d) Year

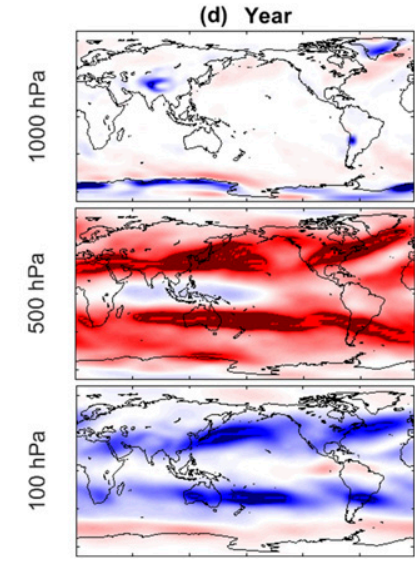

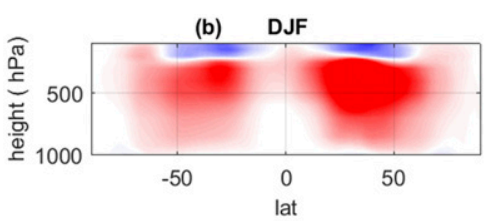

(e) DJF

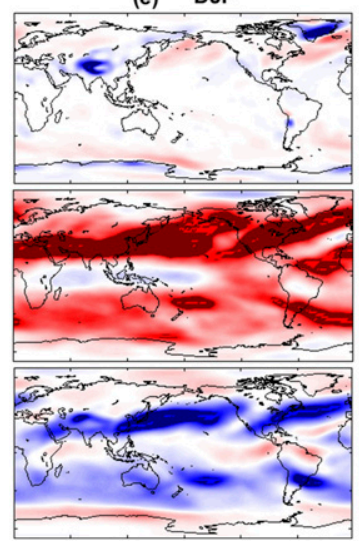

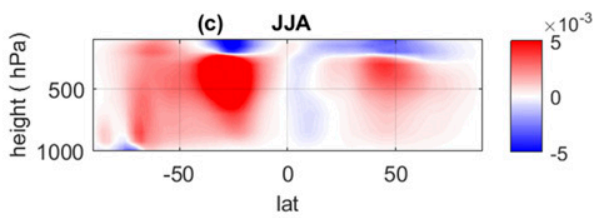

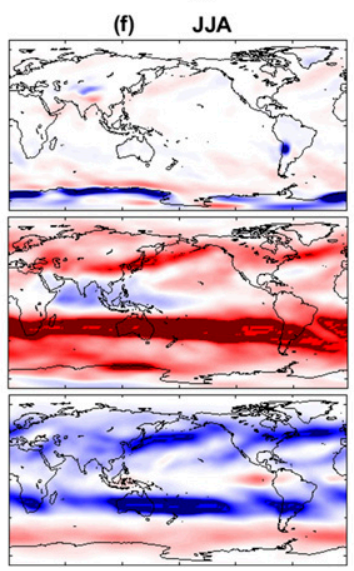

FIG. 2. As in Fig. 1, but for NCEP-NCAR reanalysis.

between a slope of $-5 / 3$ and $-7 / 5$ or between $-11 / 5$ and -3 , which are classical spectral slopes that appear in rotating stratified or quasi-2D turbulence. Moreover, the spectra are computed on a Cartesian grid, which weight oddly higher latitudes so that it is hard to trust results in the NCEP-NCAR reanalysis below $\ell=220 \mathrm{~km}$. The difference in spectra between the troposphere and stratosphere is a well-observed property of the kinetic energy spectra in the atmosphere, and several explanations have been proposed for that, some of them complementary to each other. The existence of an individual range spanning from planetary wavelengths to the edge of the subinertial range in the troposphere has been observed, for example, by Koshyk et al. (1999). They found that, above $250 \mathrm{hPa}$, there is a transition to a two-range spectrum at synoptic scales so that the subinertial range makes its way deep until about 2000-km wavelength and the spectrum exhibits a slope of $-5 / 3$. At larger wavelengths, the slope is still equal to -3 . They explain this transition with the rotational component of the kinetic energy decaying with height at a higher rate than the divergent component. Accordingly, Burgess et al. (2013) attribute this transition to the zonal mean-eddy interaction overcoming the eddy-eddy interaction in setting up the transient kinetic energy peak. Žagar et al. (2017) propose that this might be attributed to the predominant role of nonlinear inertial gravity waves. In accurate numerical simulations, Pouquet et al. (2017) have also shown that by changing the value of $f / N$, one also changes the value of the crossover between the large scales, involving an inverse energy cascade, and the small scale, involving a direct energy cascade. In the terrestrial atmosphere, the value of $f$ is practically constant, whereas $N$ can vary up to a factor of 2 between troposphere and stratosphere, thus changing the direction of the cascade.

\section{3) PROBABILITY DistribUtion FUNCTIONS OF INSTANTANEOUS LOCAL ENERGY TRANSFERS}

In addition to time average, it is also interesting to study the probability distribution function of instantaneous local energy transfers, $\mathcal{D}_{\ell}(\mathbf{u}, b)$ at a different height (pressure level), and see how it varies with scale and height. This is provided in Fig. 4 for ERA-Interim and Fig. 5 for the NCEP-NCAR reanalysis. Figures $4 \mathrm{~b}, \mathrm{~d}, \mathrm{f}$ and $5 \mathrm{~b}, \mathrm{~d}, \mathrm{f}$ show the distributions at each level for $\ell=220 \mathrm{~km}$. Figures $4 \mathrm{a}, \mathrm{b}$ and $5 \mathrm{a}, \mathrm{b}$ show the kinetic component $\mathcal{D}_{\ell}(\mathbf{u}, 0)$, Figs. $4 \mathrm{c}, \mathrm{d}$ and $5 \mathrm{c}, \mathrm{d}$ the thermodynamic component $\mathcal{D}_{\ell}^{T}$, and Figs. 4e,f and Figs. 5e,f the total $\mathcal{D}_{\ell}(\mathbf{u}, b)$. Tables S1 and S2 in the supplemental material report the values of mean, standard deviation, skewness, and kurtosis as a function of the height for the total DR indicator.

Overall, all distributions are skewed and exhibit fat tails. The sign of the skewness depends on the height: for both the total and kinetic component, it is positive in the lower troposphere and negative for $P<500 \mathrm{hPa}$, in agreement with the time averages. For the thermodynamic part, the behavior is opposite, with a negative skewness at low altitude $(P>700 \mathrm{hPa})$ and positive skewness at large altitude. In such case, the distribution is totally asymmetric and includes only positive transfer, indicating that in the higher part of the atmosphere, the 
(a)

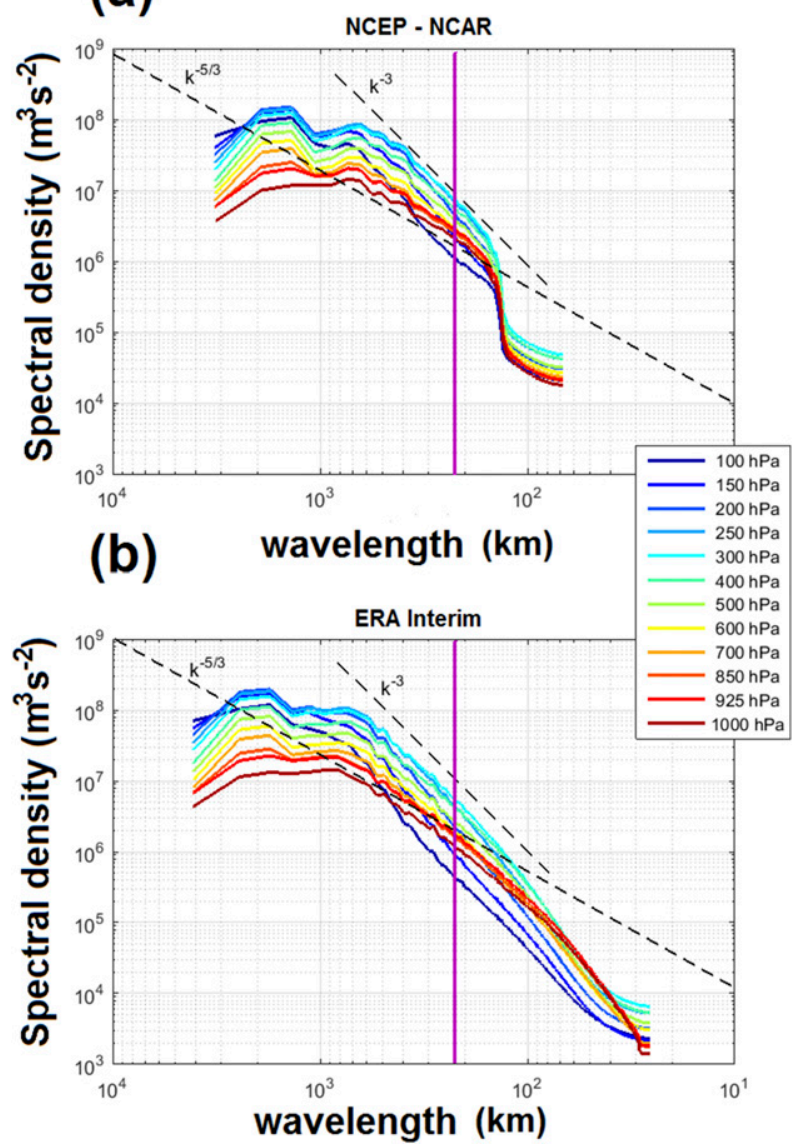

FIG. 3. (a) NCEP-NCAR reanalysis and (b) ERA-Interim. Spectra $E(k)$, where $k$ is the wavelength, computed, at each pressure level, for the horizontal velocity fields (solid lines); $-5 / 3$ and -3 slopes (dashed lines); $\ell=220 \mathrm{~km}$ (magenta vertical lines).

density fluctuations only contribute to a downscale energy transfer. Although there is agreement between the ERA-Interim and NCEP-NCAR data, the latter show fatter tails. This might be due to either the different resolution of the datasets and/or the different physical parameterizations.

Looking now at the dependence with scale at a fixed height, we see that both the kinetic and total local energy transfer display similar behavior, with a tendency to have fatter tails with decreasing scales. This means that the energy imbalance of the reanalysis is reduced when we look at motions whose characteristic scales are larger. This type of behavior, also observed in local energy transfers measured in a laboratory turbulent von Kármán flow (Saw et al. 2016), might be because, at larger scales, the atmosphere becomes more wavelike (and so less turbulent; Rhines 1979). Regarding the thermodynamical part of the transfer, the scale dependence is much milder on the positive side of the distribution and even absent in the negative part of the distribution.

\section{4) Possible INTERPRETATION}

A possible way to explain the sign of the DR indicators is to invoke the relation between baroclinic and barotropic flows and direct and inverse cascades. In Tung and Orlando (2003), it is argued that the baroclinic motions responsible for the genesis and decay of extratropical cyclones are mostly associated with direct cascades [corresponding to positive $\mathcal{D}_{\ell}(\mathbf{u}, b)$ ], while the essentially barotropic motions governing the lower-stratosphere dynamics (Salby 1996, chapter 17) are associated with an inverse energy cascade [which would correspond to negative values of $\left.\mathcal{D}_{\ell}(\mathbf{u}, b)\right]$. To check such interpretation, we have analyzed the maps of $\mathcal{D}_{\ell}(\mathbf{u})$ collected every 6 or $12 \mathrm{~h}$ depending on the datasets. They are collected for ERA-Interim in the supplemental video. Large positive and negative values of the DR indicator are found as dipoles in baroclinic eddies. When increasing the scale $\ell$ of the analysis, the tails become lighter as the local positive and negative contributions get averaged out.

\section{Discussion}

Weather and climate models do not resolve the viscous scales, which for the atmospheric motions are of order $0.1 \mathrm{~mm}$ (Priestley 1959). To date, their resolution ranges from $\simeq 2 \mathrm{~km}$ for the regional weather models to $\simeq 100 \mathrm{~km}$ for the global climate models. To correctly represent dissipation effects at a scale $\ell$, the turbulent cascade needs to be parameterized at each grid point depending on the type of motion and the geographical constraints. Despite the importance of such energy transfers, their distribution and their time and spatial behavior is known only partially through field campaigns (Lübken 1997) or by global averages (Sellers 1969; Seinfeld and Pandis 2016). This does not ensure a global coverage and does not indicate the direction of the energy transfers in the free troposphere. In this paper, we have used Duchon and Robert (2000) to compute and characterize the distribution of instantaneous and local subfilter energy transfers in the atmosphere using 3D velocity fields obtained in the NCEP-NCAR Reanalysis-1 and ERA-Interim. Those energy transfers are highly correlated with the baroclinic eddies occurring at midlatitudes and with severe tropical cyclones. Our computation of local energy transfer provides the direction of the local energy cascade at a certain scale $\ell$ in physical space. At grid resolution $\Delta x$, the value of $\mathcal{D}_{\Delta x}(\mathbf{u}, b)$ is an exact measure of the amount of energy that must be transferred to subgrid scales (positive DR contributions) or that must be injected from subgrid 

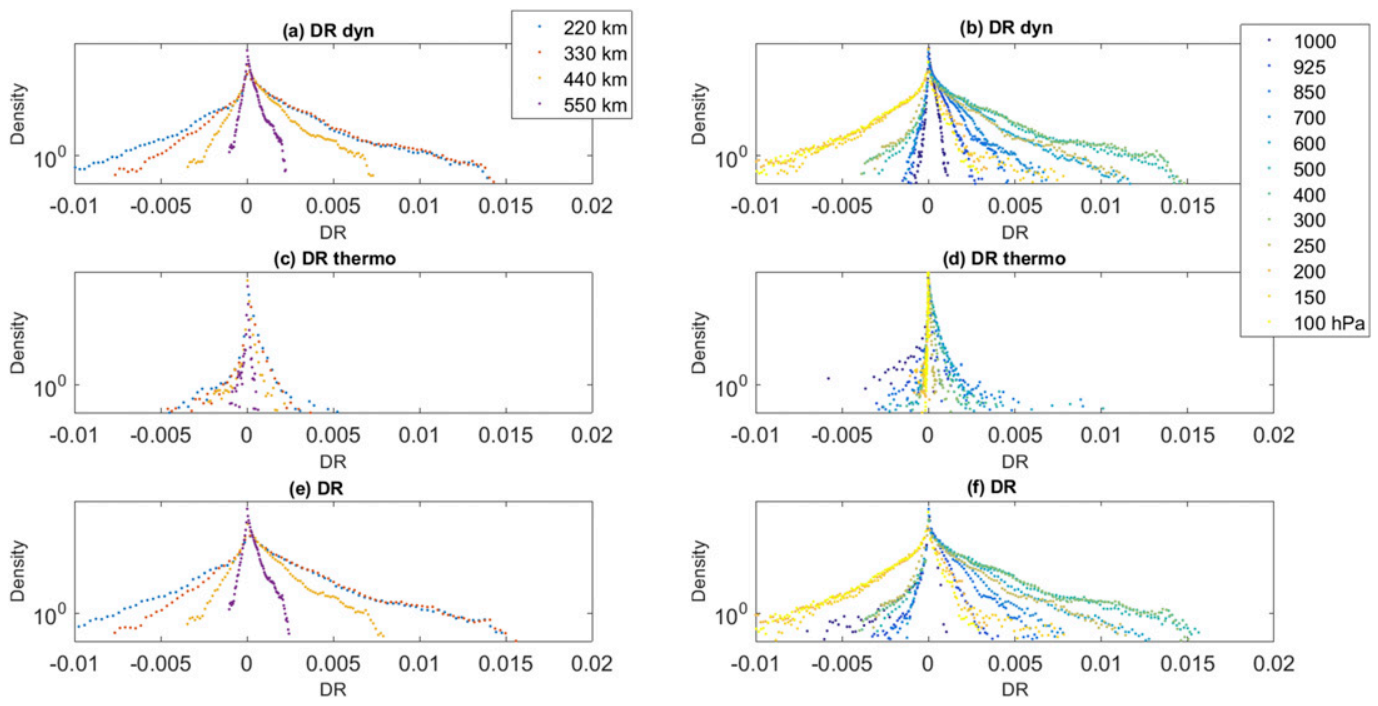

FIG. 4. Empirical $\mathcal{D}_{\ell}(\mathbf{u}, b)$ density functions for ERA-Interim against (a),(c),(e) scale $\ell$ of analysis and (b),(d),(f) height for $\ell=220 \mathrm{~km}$. (a),(b) The dynamical $\mathcal{D}_{\ell}(\mathbf{u})$ component, (c),(d) the thermodynamic $\mathcal{D}_{\ell}^{T}$ component, and (e),(f) the total $\mathcal{D}_{\ell}(\mathbf{u})$.

scales (negative contributions) in order to equilibrate energy budgets. If the simulation is perfectly resolved so that $\Delta x=\eta$, this equilibration is of course guaranteed by the contribution due to viscosity. In most cases, however, the Kolmogorov scale is not resolved, and one needs to artificially increase the viscosity so as to absorb or produce this energy flux. The information about $\mathcal{D}_{\Delta x}(\mathbf{u}, b)$ could then be used to interactively adjust the viscosity to account for the energy conservation laws in the atmosphere (Lucarini and Ragone 2011). Furthermore, the expression of $\mathcal{D}_{\ell}(\mathbf{u})$ is separable in dynamical and thermodynamic contributions. Although most of the total $\mathcal{D}_{\ell}(\mathbf{u}, b)$ contribution is due to the dynamical component, negative fluxes are found at the ground in presence of mountain ranges and sharp temperature/pressure gradients, and positive fluxes in the middle troposphere reinforce the dynamic contributions. We have also observed that extreme events such as tropical and extratropical storms are associated with large values of $\mathcal{D}_{\ell}(\mathbf{u}, b)$ even at the ground.

The quantity $\mathcal{D}_{\ell}(\mathbf{u}, b)$ could also be a proxy of the flux of energy that can be exploited in wind turbines (Miller et al. 2011, 2015). Although our analysis is performed for large-scale general circulation models, the
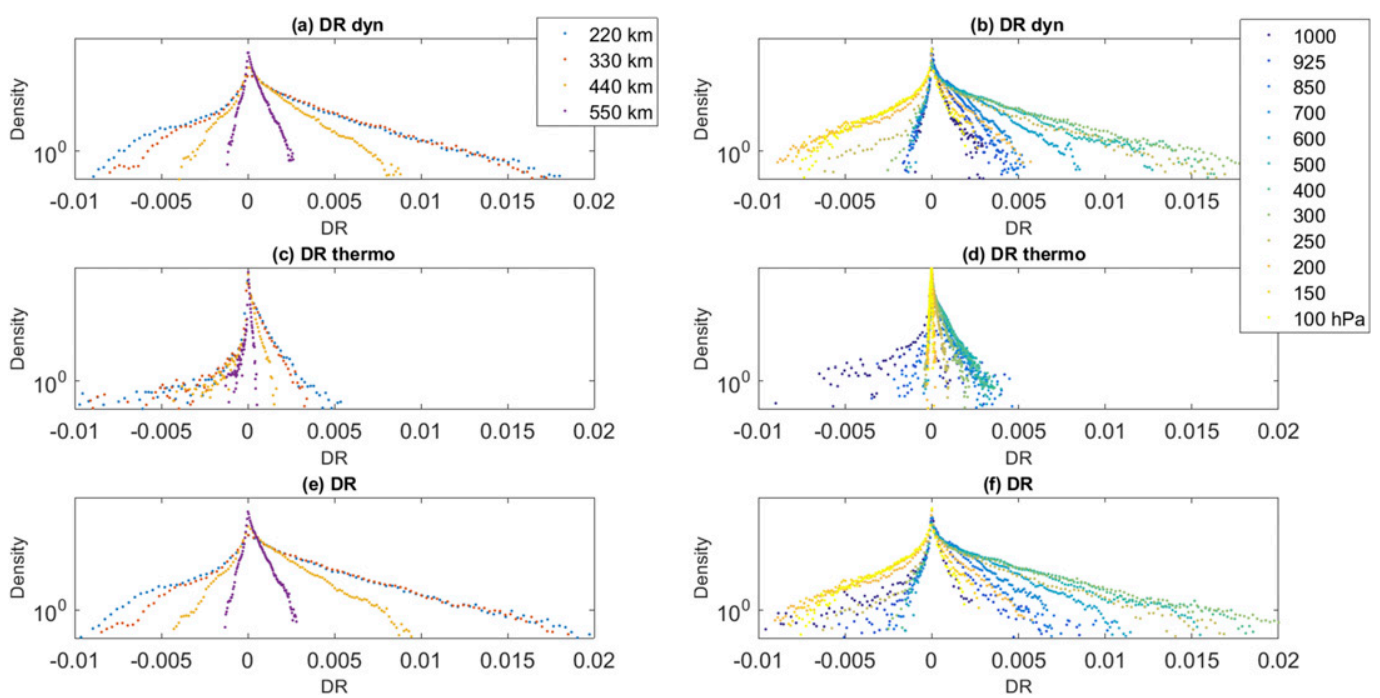

FIG. 5. As in Fig. 4, but for NCEP-NCAR reanalysis. 
Duchon and Robert (2000) formula can be applied to regional climate and weather prediction models. At smaller scales, it will be extremely interesting to analyze the relation between $\mathcal{D}_{\ell}(\mathbf{u}, b)$ and the genesis of extreme wind gusts or even tornadoes. At such scales, one could investigate the distributions of $\mathcal{D}_{\ell}(\mathbf{u}, b)$ to the instantaneous subgrid-scale dissipation obtained by field measurements (Higgins et al. 2003). It will also be worth investigating whether adaptive asymptotic methods, such as those proposed by Klein et al. (2001) or the Lagrangian scale-dependent models for the subgrid scales in large-eddy simulations (Bou-Zeid et al. 2004), afford better energy balances; that is, the spatial and temporal average of $\mathcal{D}_{\ell}(\mathbf{u}, b)$ is closer to zero.

It is evident that the resolution plays an important role in determining spurious energy fluxes by looking at the difference in the $\mathcal{D}_{\ell}(\mathbf{u}, b)$ indicator near the ground (NCEP-NCAR reanalysis vs ERA-Interim). However, it is positively surprising that the average spatial and vertical structure of the indicators is very similar in both reanalyses.

Several wave phenomena in the atmosphere-gravity and Rossby waves - are tied to these horizontal density variations and are associated with energy conversion between available potential and kinetic energy. One key question is whether this diagnostic may incorrectly assess such energy conversion as an energy transfer across scales. For future research directions, it might be worth applying the diagnostic to a simple gravity or Rossby wave model.

Acknowledgments. D. Faranda was supported by ERC Grant 338965. V. Lembo was funded by the Collaborative Research Centre TRR181 "Energy Transfers in Atmosphere and Ocean" funded by the German Research Foundation (DFG). D. F. thanks G. Messori and N. Vercauteren for useful discussions and comments on the paper.

\section{APPENDIX}

\section{Derivation of the Local Duchon-Robert Equation for Boussinesq Equations}

We start from the Boussinesq equations [Eqs. (2)] for the buoyancy perturbation and write it at two different positions, $\mathbf{x}$ and $\mathbf{x}^{\prime}$ for $b(\mathbf{x})$ and $b^{\prime}=b\left(\mathbf{x}^{\prime}\right)$ and $u(\mathbf{x})$ and $u^{\prime}=u\left(\mathbf{x}^{\prime}\right)$ :

$$
\begin{aligned}
\partial_{t} b+\partial_{j}\left(u_{j} b\right) & =-N^{2} u_{z}+\kappa \partial_{j}^{2} b, \\
\partial_{t} b^{\prime}+\partial_{j}\left(u_{j}^{\prime} b^{\prime}\right) & =-N^{2} u_{z}^{\prime}+\kappa \partial_{j}^{2} b^{\prime} .
\end{aligned}
$$

Multiplying Eq. (A1) by $b^{\prime}$ and Eq. (A2) by $b$ and adding the results, we obtain

$$
\begin{aligned}
\partial_{t}\left(b b^{\prime}\right)+b \partial_{j}\left(b^{\prime} u_{j}^{\prime}\right)+b^{\prime} \partial_{j}\left(b u_{j}\right)= & -N^{2}\left(b^{\prime} u_{z}+b u_{z}^{\prime}\right) \\
& +\kappa\left(b^{\prime} \partial_{j}^{2} b+b \partial_{j}^{2} b^{\prime}\right) .
\end{aligned}
$$

To simplify the equation, we can write the diffusive term as

$$
b^{\prime} \partial_{j}^{2} b+b \partial_{j}^{2} b^{\prime}=\partial_{j}^{2} b b^{\prime}-2 \partial_{j} b \partial_{j} b^{\prime},
$$

while the nonlinear can be written as

$$
b \partial_{j} b^{\prime} u_{j}^{\prime}+b^{\prime} \partial_{j} b u_{j}=b \delta u_{j} \partial_{j} b^{\prime}+\partial_{j} b u_{j} b^{\prime},
$$

where $\delta u_{j}=u_{j}^{\prime}-u_{j}$ as before. Considering the term $\left(u_{j}^{\prime}-u_{j}\right)\left(b^{\prime}-b\right)^{2}=\delta u_{j}(\delta b)^{2}$, it reads as

$\delta u_{j}(\delta b)^{2}=b^{\prime 2}\left(\delta u_{j}\right)+b^{2}\left(\delta u_{j}\right)-2 b^{\prime}\left(u_{j}^{\prime}-u_{j}\right) b$.

Using now the identities $\nabla_{r} \cdot(\delta \mathbf{u})=\nabla_{r} \cdot \mathbf{u}^{\prime}=0$, and after some manipulations, we have

$$
b \delta u_{j} \partial_{j} b^{\prime}=\frac{1}{2}\left\{\partial_{j}\left(b^{\prime 2} \delta u_{j}\right)-\partial_{j}\left[\delta u_{j}(\delta b)^{2}\right]\right\}+\partial_{j} b u_{j} b^{\prime} .
$$

Substituting the results from the Eqs. (A4) and (A7) and multiplying both the sides by $1 / 2$ and simplifying gives

$$
\begin{aligned}
\partial_{t} & \left(\frac{1}{2} b b^{\prime}\right)+\frac{1}{2} \partial_{j}\left[\left(u_{j} b^{\prime}\right) b+\frac{1}{2} b^{\prime 2} \delta u_{j}-\kappa \partial_{j}\left(b b^{\prime}\right)\right] \\
\quad= & \frac{1}{4} \nabla_{r} \cdot \delta \mathbf{u}(\delta b)^{2}-\kappa \partial_{j} b \partial_{j} b^{\prime}-\frac{N^{2}}{2}\left(b u_{z}^{\prime}+b^{\prime} u_{z}\right) .
\end{aligned}
$$

Applying the filter operator $G_{\ell}$, and noting $\hat{f}=f^{*} G_{\ell}$ (with the asterisk being the convolution), we get

$$
\begin{aligned}
\partial_{t}( & \left.\frac{1}{2} b \hat{b}\right)+\nabla \cdot\left[\frac{1}{2}(\mathbf{u} \hat{b}) b+\frac{1}{4}\left(\widehat{b^{2} \mathbf{u}}\right)-\frac{1}{4} \widehat{\left(b^{2}\right)} \mathbf{u}-\kappa \nabla\left(\frac{1}{2} b \hat{b}\right)\right]=-\frac{1}{4 \ell} \int d \mathbf{r}(\nabla G)_{l} \cdot \delta \mathbf{u}(r)(\delta b)^{2} \\
& -\kappa \boldsymbol{\nabla} b \cdot \nabla \hat{b}-\frac{N^{2}}{2}\left(b \hat{u}_{z}+\hat{b} u_{z}\right) .
\end{aligned}
$$


Introducing $E_{T}^{\ell}=b \hat{b} /\left(2 N^{2}\right)$, the available potential energy at scale $\ell$, and the terms

$$
\mathbf{J}_{T}^{\ell}=\left[\frac{1}{2}(\mathbf{u} \hat{b}) b+\frac{1}{4}\left(\widehat{b^{2} \mathbf{u}}\right)-\frac{1}{4} \widehat{\left(b^{2}\right)} \mathbf{u}-\kappa \nabla\left(\frac{1}{2} b \hat{b}\right)\right] / N^{2},
$$

$$
\mathcal{D}_{\ell}^{b}=\frac{1}{4 l} \int d r(\nabla G)_{l} \cdot \delta \mathbf{u}(r)(\delta b)^{2} / N^{2},
$$

we get Eq. (9) of section 2.

Now, to study the inviscid limit $\nu \rightarrow 0$, we take the limit $\ell \rightarrow 0$, and introducing the available potential energy $E_{T}=b^{2} / 2 N^{2}$, the equation finally simplifies to

$$
\begin{gathered}
\partial_{t} E_{T}+\nabla \cdot\left(\frac{1}{2} \mathbf{u} E_{T}\right)-\kappa \nabla^{2} E_{T} \\
=-b u_{z}-\mathcal{D}^{b}-\kappa(\nabla b)^{2} / N^{2},
\end{gathered}
$$

with

$$
\mathcal{D}^{b}=\lim _{l \rightarrow 0} \mathcal{D}_{\ell}^{b} .
$$

The equation for the kinetic energy has been derived in Duchon and Robert (2000) without the term due to rotation and buoyancy. For the rotation, it is straightforward to see that it only adds a term $\mathbf{u} \cdot(2 \boldsymbol{\Omega} \times \hat{\mathbf{u}})+\hat{\mathbf{u}} \cdot(2 \boldsymbol{\Omega} \times \mathbf{u})$, which vanishes, because of the symmetry of the $\times$ operator. The buoyancy adds a new term that can be simply included so that the equation for the kinetic energy can be written as

$$
\begin{gathered}
\partial_{t} E^{\ell}+\partial_{j}\left[\hat{u}_{j} E^{\ell}+\frac{1}{2}\left(u_{j} \hat{p}+\hat{u}_{j} p\right)+\frac{1}{4}\left(\widehat{u}^{2} u_{j}-\frac{1}{4} \widehat{u}^{2} u_{j}\right)\right. \\
\left.-\nu \partial_{j} E^{\ell}\right]=-\nu \partial_{j} u_{i} \partial_{j} \hat{u}_{i}-\mathcal{D}_{\ell}+\frac{1}{2}\left(b \hat{u}_{z}+\hat{b} u_{z}\right),
\end{gathered}
$$

with $\mathcal{D}_{\ell}$ being given by Eq. (6). Introducing the $\mathrm{KE}$ spatial flux,

$$
\mathbf{J}_{K}^{\ell}=\hat{\mathbf{u}} E^{\ell}+\frac{1}{2}(\mathbf{u} \hat{p}+\hat{\mathbf{u}} p)+\frac{1}{4}\left[\widehat{\left(u^{2} \mathbf{u}\right)}-\frac{1}{4} \widehat{\left(u^{2}\right)} \mathbf{u}\right]-\nu \nabla E^{\ell},
$$

we get Eq. (8) of section 2. The fact that the rotation does no enter explicitly into the kinetic energy budget is well known and because the Coriolis force does not produce energy. However, it influences the energy cascade through the energy redistribution by nonlinear mechanisms such as resonant wave interactions (Campagne et al. 2014). This process is taken into account in term $\mathcal{D}_{\ell}$ via the third-order moment.

\section{REFERENCES}

Augier, P., and E. Lindborg, 2013: A new formulation of the spectral energy budget of the atmosphere, with application to two high-resolution general circulation models. J. Atmos. Sci., 70, 2293-2308, https://doi.org/10.1175/JAS-D-12-0281.1.

_ - S. Galtier, and P. Billant, 2012: Kolmogorov laws for stratified turbulence. J. Fluid Mech., 709, 659-670, https://doi.org/ 10.1017/jfm.2012.379.

Bartello, P., 1995: Geostrophic adjustment and inverse cascades in rotating stratified turbulence. J. Atmos. Sci., 52, 4410-4428, https://doi.org/10.1175/1520-0469(1995)052<4410:GAAICI >2.0. $\mathrm{CO} ; 2$.

Beljaars, A., 1995: The parametrization of surface fluxes in largescale models under free convection. Quart. J. Roy. Meteor. Soc., 121, 255-270, https://doi.org/10.1002/qj.49712152203.

Berrisford, P., P. Kållberg, S. Kobayashi, D. Dee, S. Uppala, J. Simmons, P. Poli, and H. Sato, 2011: Atmospheric conservation properties in ERA-Interim. Quart. J. Roy. Meteor. Soc., 137, 1381-1399, https://doi.org/10.1002/qj.864.

Bou-Zeid, E., C. Meneveau, and M. B. Parlange, 2004: Large-eddy simulation of neutral atmospheric boundary layer flow over heterogeneous surfaces: Blending height and effective surface roughness. Water Resour. Res., 40, W02505, https://doi.org/ 10.1029/2003WR002475.

Brunke, M. A., Z. Wang, X. Zeng, M. Bosilovich, and C.-L. Shie, 2011: An assessment of the uncertainties in ocean surface turbulent fluxes in 11 reanalysis, satellite-derived, and combined global datasets. J. Climate, 24, 5469-5493, https://doi. org/10.1175/2011JCLI4223.1.

Burgess, B. H., A. R. Erler, and T. G. Shepherd, 2013: The troposphere-to-stratosphere transition in kinetic energy spectra and nonlinear spectral fluxes as seen in ECMWF analyses. J. Atmos. Sci., 70, 669-687, https://doi.org/10.1175/ JAS-D-12-0129.1.

Campagne, A., 2015: Cascades dénergie et turbulence dondes dans une expérience de turbulence en rotation. Ph.D. thesis, Université Paris Sud, 129 pp.

— , B. Gallet, F. Moisy, and P.-P. Cortet, 2014: Direct and inverse energy cascades in a forced rotating turbulence experiment. Phys. Fluids, 26, 125112, https://doi.org/10.1063/1.4904957.

Charney, J. G., 1971: Geostrophic turbulence. J. Atmos. Sci., 28, 1087-1095, https://doi.org/10.1175/1520-0469(1971)028<1087: GT>2.0.CO;2.

Dee, D. P., and Coauthors, 2011: The ERA-Interim reanalysis: Configuration and performance of the data assimilation system. Quart. J. Roy. Meteor. Soc., 137, 553-597, https://doi.org/ 10.1002/qj.828.

Duchon, J., and R. Robert, 2000: Inertial energy dissipation for weak solutions of incompressible Euler and Navier-Stokes equations. Nonlinearity, 13, 249-255, https://doi.org/10.1088/ 0951-7715/13/1/312.

Falkovich, G., 1992: Inverse cascade and wave condensate in mesoscale atmospheric turbulence. Phys. Rev. Lett., 69, 31733176, https://doi.org/10.1103/PhysRevLett.69.3173.

Frisch, U., 1995: Turbulence: The Legacy of A. N. Kolmogorov. Cambridge University Press, 296 pp.

Gage, K., 1979: Evidence for a $k^{-5 / 3}$ law inertial range in mesoscale two-dimensional turbulence. J. Atmos. Sci., 36, 1950-1954, https://doi.org/10.1175/1520-0469(1979)036<1950:EFALIR >2.0. $\mathrm{CO} ; 2$.

Higgins, C. W., M. B. Parlange, and C. Meneveau, 2003: Alignment trends of velocity gradients and subgrid-scale fluxes in the 
turbulent atmospheric boundary layer. Bound.-Layer Meteor., 109, 59-83, https://doi.org/10.1023/A:1025484500899.

Holton, J. R., and G. J. Hakim, 2012: An Introduction to Dynamic Meteorology. 5th ed. International Geophysics Series, Vol. 88, Academic Press, 552 pp.

Kalnay, E., and Coauthors, 1996: The NCEP/NCAR 40-Year Reanalysis Project. Bull. Amer. Meteor. Soc., 77, 437-471, https:// doi.org/10.1175/1520-0477(1996)077<0437:TNYRP>2.0.CO;2.

Kitamura, Y., and Y. Matsuda, 2006: The $k_{H}^{-3}$ and $k_{H}^{-5 / 3}$ energy spectra in stratified turbulence. Geophys. Res. Lett., 33, L05809, https://doi.org/10.1029/2005GL024996.

Klein, R., N. Botta, T. Schneider, C.-D. Munz, S. Roller, A. Meister, L. Hoffmann, and T. Sonar, 2001: Asymptotic adaptive methods for multi-scale problems in fluid mechanics. Practical Asymptotics, Springer, 261-343.

Kolmogorov, A. N., 1941: Dissipation of energy in locally isotropic turbulence. Dokl. Akad. Nauk SSSR, 32, 16-18.

Koshyk, J. N., B. A. Boville, K. Hamilton, E. Manzini, and K. Shibata, 1999: Kinetic energy spectrum of horizontal motions in middle-atmosphere models. J. Geophys. Res., 104, 27 177-27 190, https://doi.org/10.1029/1999JD900814.

Kuzzay, D., D. Faranda, and B. Dubrulle, 2015: Global vs local energy dissipation: The energy cycle of the turbulent von Kármán flow. Phys. Fluids, 27, 075105, https://doi.org/10.1063/ 1.4923750 .

Levich, E., and E. Tzvetkov, 1985: Helical inverse cascade in threedimensional turbulence as a fundamental dominant mechanism in mesoscale atmospheric phenomena. Phys. Rep., 128, 1-37, https://doi.org/10.1016/0370-1573(85)90036-5.

Lilly, D. K., 1983: Stratified turbulence and the mesoscale variability of the atmosphere. J. Atmos. Sci., 40, 749-761, https://doi.org/ 10.1175/1520-0469(1983)040<0749:STATMV>2.0.CO;2.

Lindborg, E., 2005: The effect of rotation on the mesoscale energy cascade in the free atmosphere. Geophys. Res. Lett., 32, L01809, https://doi.org/10.1029/2004GL021319.

, 2006: The energy cascade in a strongly stratified fluid. J. Fluid Mech., 550, 207-242, https://doi.org/10.1017/S0022112005008128.

- and J. Y. N. Cho, 2001: Horizontal velocity structure functions in the upper troposphere and lower stratosphere: 2. Theoretical considerations. J. Geophys. Res., 106, $10233-$ 10 241, https://doi.org/10.1029/2000JD900815.

Lorenz, E. N., 1955: Available potential energy and the maintenance of the general circulation. Tellus, 7, 157-167, https:// doi.org/10.3402/tellusa.v7i2.8796.

Lovejoy, S., and D. Schertzer, 2010: Towards a new synthesis for atmospheric dynamics: Space-time cascades. Atmos. Res., 96, 1-52, https://doi.org/10.1016/j.atmosres.2010.01.004.

Lübken, F.-J., 1997: Seasonal variation of turbulent energy dissipation rates at high latitudes as determined by in situ measurements of neutral density fluctuations. J. Geophys. Res., 102, 13 441-13 456, https://doi.org/10.1029/97JD00853.

Lucarini, V., and F. Ragone, 2011: Energetics of climate models: Net energy balance and meridional enthalpy transport. Rev. Geophys., 49, RG1001, https://doi.org/ 10.1029/2009RG000323.

Miller, L. M., F. Gans, and A. Kleidon, 2011: Estimating maximum global land surface wind power extractability and associated climatic consequences. Earth Syst. Dyn., 2, 1-12, https://doi.org/10.5194/esd-2-1-2011.

— N. A. Brunsell, D. B. Mechem, F. Gans, A. J. Monaghan, R. Vautard, D. W. Keith, and A. Kleidon, 2015: Two methods for estimating limits to large-scale wind power generation.
Proc. Natl. Acad. Sci. USA, 112,11 169-11 174, https://doi.org/ 10.1073/pnas.1408251112.

Miyakoda, K., and J. Sirutis, 1986: Manual of the e-physics. Geophysical Fluid Dynamics Laboratory Rep., 122 pp.

Nastrom, G., and K. S. Gage, 1985: A climatology of atmospheric wavenumber spectra of wind and temperature observed by commercial aircraft. J. Atmos. Sci., 42, 950-960, https:// doi.org/10.1175/1520-0469(1985)042<0950:ACOAWS $>2.0$. $\mathrm{CO} ; 2$.

Parrish, D. F., and J. C. Derber, 1992: The National Meteorological Center's spectral statistical-interpolation analysis system. Mon. Wea. Rev., 120, 1747-1763, https://doi.org/10.1175/15200493(1992) $120<1747$ :TNMCSS $>2.0$.CO;2.

Peng, J., L. Zhang, and J. Guan, 2015: Applications of a moist nonhydrostatic formulation of the spectral energy budget to baroclinic waves. Part I: The lower-stratospheric energy spectra. J. Atmos. Sci., 72, 2090-2108, https://doi.org/10.1175/ JAS-D-14-0306.1.

Pouquet, A., and R. Marino, 2013: Geophysical turbulence and the duality of the energy flow across scales. Phys. Rev. Lett., 111, 234501, https://doi.org/10.1103/PhysRevLett.111.234501.

$\longrightarrow,-$ - P. D. Mininni, and D. Rosenberg, 2017: Dual constantflux energy cascades to both large scales and small scales. Phys. Fluids, 29, 111108, https://doi.org/10.1063/1.5000730.

Priestley, C. H. B., 1959: Turbulent Transfer in the Lower Atmosphere. University of Chicago Press, $130 \mathrm{pp}$.

Rhines, P. B., 1979: Geostrophic turbulence. Annu. Rev. Fluid Mech., 11, 401-441, https://doi.org/10.1146/annurev.fl.11. 010179.002153 .

Salby, M. L., 1996: Fundamentals of Atmospheric Physics. International Geophysics Series, Vol. 61, Academic Press, 627 pp.

Saw, E.-W., D. Kuzzay, D. Faranda, A. Guittonneau, F. Daviaud, C. Wiertel-Gasquet, V. Padilla, and B. Dubrulle, 2016: Experimental characterization of extreme events of inertial dissipation in a turbulent swirling flow. Nat. Commun., 7, 12466, https://doi.org/10.1038/ncomms12466.

Schertzer, D., S. Lovejoy, F. Schmitt, Y. Chigirinskaya, and D. Marsan, 1997: Multifractal cascade dynamics and turbulent intermittency. Fractals, 5, 427-471, https://doi.org/10.1142/ S0218348X97000371.

Seinfeld, J. H., and S. N. Pandis, 2016: Atmospheric Chemistry and Physics: From Air Pollution to Climate Change. John Wiley and Sons, $1152 \mathrm{pp}$.

Sellers, W. D., 1969: A global climatic model based on the energy balance of the earth-atmosphere system. J. Appl. Meteor., 8, 392-400, https://doi.org/10.1175/1520-0450(1969)008<0392: $\mathrm{AGCMBO}>2.0 . \mathrm{CO} ; 2$.

Tung, K. K., and W. W. Orlando, 2003: The $k^{-3}$ and $k^{-5 / 3}$ energy spectrum of atmospheric turbulence: Quasigeostrophic twolevel model simulation. J. Atmos. Sci., 60, 824-835, https:// doi.org/10.1175/1520-0469(2003)060<0824:TKAKES $>2.0$. $\mathrm{CO} ; 2$.

Viterbo, P., and A. C. Beljaars, 1995: An improved land surface parameterization scheme in the ECMWF model and its validation. J. Climate, 8, 2716-2748, https://doi.org/10.1175/15200442(1995)008<2716:AILSPS>2.0.CO;2.

changes to the albedo of the boreal forests in the presence of snow. J. Geophys. Res., 104, 27 803-27 810, https://doi.org/ 10.1029/1998JD200076.

Žagar, N., D. Jelić, M. Blaauw, and P. Bechtold, 2017: Energy spectra and inertia-gravity waves in global analyses. J. Atmos. Sci., 74, 2447-2466, https://doi.org/10.1175/JAS-D-16-0341.1. 